\title{
EDITORIAL
}

\section{Striking the right balance}

\author{
The recent report from the Institute of Medicine on the US systems for ensuring drug safety \\ emphasized deficiencies in post-approval assessment of drug risks. As the drug safety \\ debate progresses, the importance of assessing drug risks and benefits together should not \\ be forgotten, and nor should the harm that can be caused by undue delay of new therapies.
}

One hundred years ago this year, the US FDA was created. Since then, the regulation of pharmaceuticals in the United States has evolved considerably - most significantly in 1938 and 1962. The Federal Food, Drug and Cosmetic Act of 1938, which provides the basic structure for drug regulation in the United States, introduced the requirement for passive approval by the FDA based on evidence that a drug was safe for its intended purpose. In 1962, the most pivotal amendments to this Act were made: in order for a drug to be marketed, a company had to provide evidence of both its efficacy and safety, and an affirmative approval from the FDA was now required.

The regulatory changes of 1938 and 1962 were both part of the response to tragic events: deaths from sulphanilamide dissolved in antifreeze in the first instance, and birth defects caused by thalidomide in the second. In both cases, the system of drug regulation - and its ability to ensure drug safety in particular - was considered to be seriously flawed, and changes were made to strengthen it with the aim of preventing such tragedies.

Now, four decades on, there is again a major crisis of confidence in the ability of the FDA to ensure drug safety, this time caused by high-profile drug withdrawals, most notably Vioxx in 2004. In response, the FDA commissioned a report from the Institute of Medicine (IOM) to assess the US drug safety system. The IOM assembled a committee of independent experts (see page 888 for an interview with one of them, Alasdair Breckenridge, the chairman of the UK's regulatory agency), who, after a year and half of deliberations and interviews with various stakeholders including the pharma industry, published their report on September 21.

A key theme of the 25 recommendations in the report is the concept of a life-cycle approach to the assessment of the risks and benefits of new drugs, recognizing that FDA approval of a drug should not mark the end of efforts to more fully understand them. Indeed, given the limitations of pre-approval trials - which are generally conducted in controlled settings and typically involve more carefully selected populations than those that subsequently receive the drug - an FDA approval decision cannot be expected to represent a lifetime guarantee that a drug will be safe and effective.
But at present, the committee concluded, the US systems for assessing drug safety post-approval are outdated, inefficient and under-resourced (see page 884). Among a wide range of other measures, the report therefore recommended that the FDA's Center for Drug Evaluation and Research develop and continually improve a systematic approach to risk:benefit analysis for use pre- and postapproval, and that the FDA should have the legal authority to require such post-marketing studies as are deemed necessary to monitor safety for a particular product.

For industry, the prospect of having to invest in further studies may not be appealing. But industry, as well as the FDA, will presumably be relieved that the report has not endorsed one major proposal that has been called for recently: the creation of separate regulatory centres responsible for pre-market and post-market drug review. The potential danger of such a move is clear: undermining the ability to assess the delicate balance between risk and benefit.

Indeed, there have been suggestions lately that greater regulatory caution in recent months might have stymied the development of novel drugs ${ }^{2}$. Although delay in approving a drug can certainly prevent harm owing to its use, harm caused by the lack of availability of an effective drug should also be considered. On this topic, another landmark act in US drug regulation in the past century - the Prescription Drug User Fee Act (PDUFA), through which industry provides substantial funding to enhance the FDA's pre-approval activities - comes up for its third renewal next year. The FDA would clearly need further funding to implement the IOM recommendations, and PDUFA IV might well be expected to play a part here. Nevertheless, for the benefit of public health, and not just that of the pharma industry, it is important that when increasing emphasis on enhancing drug safety in the future, recent efforts involving the FDA to improve the process of bringing drugs to market are not neglected. Greater investment of public resources in the FDA's activities overall is needed to tackle the challenges of its second century.

\footnotetext{
. The Future of Drug Safety: Promoting and Protecting the Health of the Public [online], < http://www.iom.edu/CMS/3793/26431/37329. aspx > (2006).

2. Pollack, A. New sense of caution at F.D.A. New York Times (29 Sep 2006).
} 